\title{
Specify Cause of Death
}

National Cancer Institute

\section{Source}

National Cancer Institute. Specify Cause of Death. NCI Thesaurus. Code C158794.

A directive to specify the cause of death. 